\title{
REMARKS ON GEODESICS
}

\author{
PHILIP HARTMAN
}

\begin{abstract}
For a $C^{1}$ Riemann metric, we investigate questions concerning "curvature" and " $C^{1}$ dependence of geodesics on initial conditions".
\end{abstract}

1. Statement of results. In what follows, it is assumed that the coefficients of the positive definite Riemann metric

$$
d s^{2}=g_{i j}(u) d u^{i} d u^{j}, \quad \text { where } g_{i j}=g_{j i},
$$

are of class $C^{1}$ on an open $u=\left(u^{1}, \ldots, u^{n}\right)$ neighborhood of $u=0$. The initial value problem for geodesics of $(1.1)$ is

$$
\begin{gathered}
u^{i^{\prime \prime}}+\Gamma_{j k}^{i}(u) u^{j^{\prime}} u^{k^{\prime}}=0, \quad i=1, \ldots, n, \\
u(0)=u_{0}, \quad u^{\prime}(0)=u_{0}^{\prime}(\neq 0),
\end{gathered}
$$

where the Christoffel symbols $\Gamma_{j k}^{i}=\Gamma_{k j}^{i}$ are, of course, continuous. We shall say that (1.1) [or (1.2)] has property (*) at $u=0$ when the following holds:

(*) There exists a neighborhood $U$ of $u=0$ such that (1.2) has a unique solution $u=u(s)=u\left(s, u_{0}, u_{0}^{\prime}\right)$ and $u\left(s, u_{0}, u_{0}^{\prime}\right), u^{\prime}\left(s, u_{0}, u_{0}^{\prime}\right)$ are of class $C^{\prime}$ (as functions of their $2 n+1$ arguments on their domain of definition) for arbitrary $u_{0} \in U$ and $u_{0}^{\prime} \neq 0$.

THEOREM (II) of [4, p. 278] (cf. also [5] or [6, Theorem 6.1, p. 104]) states that if $f(s, x)$ is continuous on an open $(s, x)=\left(s, x^{1}, \ldots, x^{m}\right)$ set $E$, then a necessary and sufficient condition for

$$
x^{\prime}=f(s, x), \quad x\left(f_{0}\right)=x_{0}
$$

to have a unique solution $x=\eta\left(s, s_{0}, x_{0}\right)$, which is of class $C^{1}$ as a function of its $m+2$ arguments, is that, in the vicinity of every point $\left(s_{0}, x_{0}\right) \in E$, there exists a continuous, nonsingular $m \times m$ matrix $A(s, x)$ such that

$$
\omega=A(s, x)[d x-f(s, x) d s]
$$

has a continuous exterior derivative. (A 1 -form $\omega$ with continuous coefficients is said to have a continuous exterior derivative if there exists a 2 -form, say $d \omega$, with continuous coefficients for which Stokes' formula $\int_{\partial \Sigma} \omega=\iint_{\Sigma} d \omega$ holds for all admissible 2-surfaces $\Sigma$; cf., e.g., [5] or [6, pp. 101-104].)

It was pointed out in [4, pp. 282-284] (cf. [6, Exercise 6.2, pp. 106, 563]) that the result just quoted implies that (1.1) has property $(*)$ if (1.1) has a continuous

Received by the editors February 4, 1983.

1980 Mathematics Subject Classification. Primary 53B20; Secondary 34A10. 
Riemann curvature tensor, that is, if the 1 -forms

$$
\omega_{k 0}^{i}=\Gamma_{k j}^{i}(u) d u^{j} \text { for } i, k=1, \ldots, n,
$$

have continuous exterior derivatives. This follows by letting $x=(u, v)=\left(u, u^{\prime}\right)$ be a $2 n$-vector, identifying (1.3) with (1.2), that is, with the first order system

$$
u^{i^{\prime}}=v^{i}, \quad v^{i^{\prime}}=-\Gamma_{j k}^{i}(u) v^{j} v^{k} \quad \text { for } i=1, \ldots, n,
$$

and choosing $A$ in (1.4) to be the $2 n \times 2 n$ matrix

$$
A=\left(\begin{array}{cc}
I & 0 \\
B & I
\end{array}\right)
$$

where $B=B(u, v)$ is the $n \times n$ matrix $\left(b_{i j}\right)=\left(-v^{k} \Gamma_{k j}^{i}(u)\right)$ for $i, j=1, \ldots, n$.

In view of the theorem concerning (1.3) quoted above, it is natural to ask if (1.1) has property (*) if and only if it has a continuous Riemann curvature tensor. The answer is in the negative; cf. Proposition 1.2. The considerations below would seem to indicate that there is no reasonable geometric condition on (1.1) or (1.2) which is necessary and sufficient for (*). The analytic condition provided by $[4$, (II)] is that on a neighborhood of $(s, u, v)=\left(0,0, v_{0}\right)$ there exist continuous $n \times n$ matrices $\left(b_{i j}(s, u, v)\right),\left(c_{i j}(s, u, v)\right)$ such that $\operatorname{det}\left(c_{i j}\right) \neq 0$ and

$$
\omega_{i}=b_{i j}\left[d u^{j}-v^{j} d s\right]+c_{i j}\left[d v^{j}+\Gamma_{p q}^{j} v^{p} v^{q} d s\right], \quad i=1, \ldots, n,
$$

have continuous exterior derivatives. We first note that the geometric sufficient condition involving (1.5) can be generalized as follows.

THEOREM 1.1. A sufficient condition for (1.1) to have property (*) at $u=0$ is that, on an open neighborhood $U$ of $u=0$, there exist continuous functions $\Delta_{j k}^{i}(u)=-\Delta_{k j}^{i}(u)$ for $i, j, k=1, \ldots, n$ such that the connection $\Gamma_{j k}^{i}+\Delta_{j k}^{i}$ has a continuous curvature tensor, that is, the 1-forms

$$
\omega_{k}^{i}=\left[\Gamma_{k j}^{i}(u)+\Delta_{k j}^{i}(u)\right] d u^{j} \quad \text { for } i, k=1, \ldots, n,
$$

have continuous exterior derivatives on $U$.

This follows by choosing (1.7) with $B=\left(b_{i j}(u, v)\right), b_{i j}=-v^{k}\left[\Gamma_{k j}^{i}(u)+\Delta_{k j}^{i}(u)\right]$. It also follows from the fact that (*) does not depend on the $g_{i j}$ but rather on the $\Gamma_{j k}^{i}$, and (1.2) is not altered if $\Gamma_{j k}^{i}$ is replaced by $\Gamma_{j k}^{i}+\Delta_{j k}^{i}$.

The sufficient condition involving (1.9) is more general than the case $\Delta_{j k}^{i}=0$, i.e., the condition involving (1.5). In fact, we shall give an example proving the following:

Proposition 1.2. There exist $C^{1}$ metrics (1.1), with $n=2$, satisfying the conditions of Theorem 1.1, hence having property (*), but not having a continuous curvature.

The sufficient condition for (*) given by Theorem 1.1 is not necessary.

THEOREM 1.3. In Theorem 1.1, the requirement that (1.9) have a continuous exterior derivative on $U$ can be relaxed as follows: there exists a closed set $C$ such that (1.9) is $S$-Lipschitz continuous on $U$ and has a continuous exterior derivative on $U \backslash C$ and, for every geodesic arc $\Gamma$ of (1.1), the intersection $\Gamma \cap C$ has s-measure 0. 
This follows from Theorem $\mathrm{A}$ in the Appendix which is a generalization of part of (II) in [4] (i.e., [6, Theorem 6.1, p. 104]). It is a corrected version of (III) in [7] which is false. Theorem 1.3 is more general than Theorem 1.1 in the following sense.

Proposition 1.4. (i) There exist metrics (1.1) of class $C^{1}$, with $n=2$, satisfying the conditions of Theorem 1.3 with $\Delta_{j k}^{i} \equiv 0$, hence having property (*), but not satisfying the conditions of Theorem 1.1 for any choice of the continuous functions $\Delta_{j k}^{i}(u)=$ $-\Delta_{k j}^{i}(u)$. (ii) Theorem 1.3 may be false even if there is only one geodesic arc $\Gamma_{0}$ such that $\Gamma_{0} \cap C$ has a positive (arclength) measure.

2. Proof of Proposition 1.2. Let $u=\left(u^{1}, u^{2}\right)=(x, y)$. An example (1.1) proving Proposition 1.2 will be chosen in the conformal form

$$
d s^{2}=e^{z(x, y)}\left(d x^{2}+d y^{2}\right),
$$

where $z(x, y)$ is of class $C^{1}$ for small $|x|,|y|$. Note that when $z \in C^{2}$, the curvature of $(2.1)$ is

$$
K=-\frac{1}{2} e^{-z}\left(z_{x x}+z_{y y}\right) .
$$

The function $z(x, y)$ will be furnished by the following:

On $D=\left\{(x, y): x^{2}+y^{2} \leqslant 1\right\}$, there exists a continuous function $p(x, y)$ with the property that the "Poisson" equation

$$
2 z_{x x}+z_{y y}=p(x, y)
$$

has a $C^{1}(D)$-solution $z$ such that $z_{x y}$ exists and is continuous, but $z_{x x}, z_{y y}$ do not exist at $(x, y)=(0,0)$.

This is clear from the criteria in [9] for the existence and continuity of second order partials of logarithmic potentials. (2.3) is understood in the $L^{2}$ sense.

Suppose, if possible, that the conformal metric (2.1) has a continuous curvature $K(x, y)$ on some open set $U$ in $D$. Then (2.2) holds in a (local) $L^{2}$ sense on $U$; cf. [6, Lemma 5.1, p. 102 and Exercise 6.2, p. 106]. Thus (2.2) and (2.3) imply that the $L^{2}$ functions $z_{x x}, z_{y y}$ are equal (almost everywhere) to continuous functions on $U$. But this is not the case.

It remains to show that (2.1) satisfies the conditions of Theorem 1.1 The differential equations for the geodesics of (2.1) are

$$
2 x^{\prime \prime}+z_{k} x^{\prime 2}+2 z_{y} x^{\prime} y^{\prime}-z_{x} y^{\prime 2}=0, \quad 2 y^{\prime \prime}-z_{y} x^{\prime 2}+2 z_{x} x^{\prime} y^{\prime}+z_{y} y^{\prime 2}=0 .
$$

Hence, the forms (1.9) are given by

$$
\begin{array}{ll}
2 \omega_{1}^{1}=z_{x} d x+\left(z_{y}+2 \Delta_{12}^{1}\right) d y, & 2 \omega_{2}^{1}=\left(z_{y}+2 \Delta_{21}^{1}\right) d x-z_{x} d y, \\
2 \omega_{1}^{2}=-z_{y} d x+\left(z_{x}+2 \Delta_{12}^{2}\right) d y, & 2 \omega_{2}^{2}=\left(z_{x}+2 \Delta_{21}^{2}\right) d x+z_{y} d y,
\end{array}
$$

where $\Delta_{j j}^{i}=0$. Let $\Delta_{12}^{1}=-\Delta_{21}^{1}=z_{y} / 4$ and $\Delta_{12}^{2}=-\Delta_{21}^{2}=z_{x} / 2$, so that

$$
\begin{array}{ll}
4 \omega_{1}^{1}=2 z_{x} d x+3 z_{y} d y, & 4 \omega_{2}^{1}=z_{y} d x-2 z_{x} d y, \\
2 \omega_{1}^{2}=-z_{y} d x+2 z_{x} d y, & 4 \omega_{2}^{2}=z_{x} d x+2 z_{y} d y .
\end{array}
$$

These forms have continuous exterior derivatives since $d\left(z_{x} d x\right)=-z_{x y} d x d y$, $d\left(z_{y} d y\right)=z_{x y} d x d y$, and $d\left(z_{y} d x-2 z_{x} d y\right)=-p(x, y) d x d y$. 
3. Proof of Proposition 1.4. Let $u=\left(u^{1}, u^{2}\right)=(x, y)$ and let (1.1) be the metric

$$
d s^{2}=d x^{2}+f(x) d y^{2}
$$

where $f(x) \equiv f_{0}(x)=1+x+x|x|$ or $f(x) \equiv f_{1}(x)=1+x|x|$, and $2|x|<1$. $\mathrm{Up}$ to the addition of a continuous function of $x$, the curvature of (3.1) is $f_{x x} / 2 f=(\operatorname{sgn} x) / f(x)$. Thus (3.1) has a bounded curvature, continuous except on the (nonempty) set $C=\{(x, y): x=0\}$. The differential equations for the geodesics of (3.1) are

$$
2 x^{\prime \prime}-f_{x}(x) y^{\prime 2}=0, \quad\left(f(x) y^{\prime}\right)^{\prime}=0 .
$$

Hence, on any geodesic, $x^{\prime \prime} \geqslant 0$ and either $y^{\prime} \equiv 0$ or $y^{\prime} \neq 0$. In the first of these cases, we have $y^{\prime} \equiv 0$ and $x^{\prime} \neq 0$, so that the corresponding geodesic meets $C$ in one point.

In the case where $y^{\prime} \neq 0$ and $f=f_{0}(x)$ with $f_{x}(x)=1+2|x|>0$, we have $x^{\prime \prime}>0$, so that the geodesic meets $C$ in at most two points. Hence, (3.1) with $f=f_{0}$ satisfies the conditions of Theorem 1.3 with $\Delta_{j k}^{i}=0$.

In the case $f=f_{1}(x)$ with $f_{x}(0)=0$, the line $C$ is a geodesic and all other geodesics meet $C$ in at most two points. Hence, except for the geodesic $\Gamma_{0}=C$, the conditions of Theorem 1.3 are satisfied. It was shown, however, in [8, pp. 329-330], that for (3.1) with $f=f_{1}$, the geodesics are uniquely determined by initial conditions but are not dependent on them in a $C^{1}$ manner. This example proves Proposition 1.4(ii).

In order to complete the proof of (i), it must be shown that (3.1) with $f=f_{0}$ does not satisfy the conditions of Theorem 1.1. If it does, then there exists a continuous function $g(x, y)=2 \Delta_{21}^{1}$ such that

$$
2 \omega_{2}^{1}=g d x-f_{x} d y=g d x-(1+2|x|) d y
$$

has a continuous exterior derivative, say, $-p(x, y) d x d y$. It follows that, for $x \neq 0, g$ has a continuous partial derivative $g_{y}$ and

$$
g_{y}=p(x, y)-f_{x x}(x)=p(x, y)-2 \operatorname{sgn} x \text { for } x \neq 0
$$

cf. [6. Exercise 5.2, pp. 104, 562]. Hence, for $x \neq 0$,

$$
g(x, y)=-2 y \operatorname{sgn} x+\int_{0}^{y} p(x, t) d t+g(x, 0) .
$$

But a function of this form cannot be continuous for small $|x|,|y|$.

Appendix. Theorem (II) of [4] (i.e., [6, Theorem 6.1, p. 104]) gives necessary and sufficient conditions in order that

$$
x^{\prime}=f(s, x), \quad x\left(s_{0}\right)=x_{0},
$$

has a unique solution $x=\eta\left(s, s_{0}, x_{0}\right)$ which is of class $C^{1}$ as function of all its arguments. Here we give a "generalization" of the sufficiency part which implies Theorem 1.3 above.

TheOREM A. Let $f(s, x)$ be continuous on an open $(s, x)=\left(s, x^{1}, \ldots, x^{m}\right)$ set $U$ with the property that there exists a closed set $C$ and a continuous nonsingular $m \times m$ 
matrix $A(s, x)$ on $U$ such that: (i) the 1-form

$$
\omega=A(s, x)[d x-f(x, x) d x]
$$

is Lipschitz continuous on $U$ (or is merely S-Lipschitz continuous on $U$; cf. [6, pp. 107-109]) and has a continuous exterior derivative on $U \backslash C$; and (ii) every solution arc $(s, x(s))$ of (1) meets $C$ in a set of $s$-measure 0 . Then the initial value problem (1) has a unique solution $x=\eta\left(s, s_{0}, x_{0}\right)$ which is of class $C^{1}$ on its $(m+2)$-dimensional domain of existence.

The simplest proof of Theorem A is obtained by modifying the arguments of $[6, p$. 113] in the proof of Theorem 6.1. The considerations below make clear what modifications are necessary. In order to avoid repeating the entire proof, we give a slightly different argument using Theorem 6.1 and Corollary 6.1 of [6, pp. 104-105].

Proof of Theorem A. According to [5] (cf. [6, Theorem 8.1, p. 109]), the fact that (2) is $S$-Lipschitz continuous implies (1) has a unique solution $x=\eta\left(s, s_{0}, x_{0}\right)$ which is locally uniformly Lipschitz continuous on its domain of existence, say $\Omega$.

The conditions on (2) imply there exists a continuous, bounded $m \times m$ matrix function $F(s, x)$ on $U \backslash C$ with the property that if $\left(s_{0}, x_{0}\right) \notin C$, then $y=\partial \eta / 2 x_{0}^{k}$, $k=1, \ldots, m$, exists on any $s$-interval containing $s_{0}$ along which $(s, x)=$ $\left(s, \eta\left(s, s_{0}, x_{0}\right)\right) \notin C$ and is a solution of

$$
[A(s, \eta) y]^{\prime}=F(s, \eta) y, \quad y\left(s_{0}\right)=e_{k},
$$

where $e_{k}=\left(e_{k 1}, \ldots, e_{k m}\right), e_{k j}=0$ for $k \neq j$ and $e_{k k}=1$, and $\eta=\eta\left(s, s_{0}, x_{0}\right)$; cf. [4, 5] or [6, Corollary 6.1, p. 105].

The matrix function $A(s, \eta)$ is continuous for $\left(s, s_{0}, x_{0}\right) \in \Omega$ and is nonsingular. Also $F(s, \eta)$ is a bounded continuous function of $\left(s, s_{0}, x_{0}\right)$ on its domain of definition. For fixed $\left(s_{0}, x_{0}\right)$, the set of $s$-values for which $F(s, \eta)$ is not defined is of measure 0 . It follows that, whether or not $\left(s_{0}, x_{0}\right) \in C$, there exists a unique solution $y=y(s)=y\left(s, s_{0}, x_{0}\right)$ of (3) (in the sense that $y$ is an absolutely continuous function of $s$ satisfying (4) almost everywhere) which is a continuous function of $\left(s, s_{0}, x_{0}\right)$ defined on the domain $\Omega$ of existence of $\eta\left(s, s_{0}, x_{0}\right)$. This can be verified by a modification of standard arguments involving linear differential equations; $c f$. , e.g., [6, proof of Corollary 4.1, p. 55].

Since $\eta\left(s, s_{0}, x_{0}\right)$ is locally uniformly Lipschitz continuous, the partial derivative $\partial \eta / \partial x_{0}^{k}$ exists except on an $\left(s, s_{0}, x_{0}\right)$-set of $(m+2)$-dimensional measure 0 . This partial derivative $\partial \eta / \partial x_{0}^{k}$ has an extension to the continuous function $y\left(s, s_{0}, x_{0}\right)$ on $\Omega$. Hence, $\partial \eta / \partial x_{0}^{k}$ exists and is continuous on $\Omega$.

In a standard way, it follows that $\partial \eta / \partial s_{0}$ exists and is the continuous function $\partial \eta / \partial s_{0}=-\Sigma\left(\partial \eta / \partial x_{0}^{k}\right) f^{k}\left(s_{0}, x_{0}\right)$ on $\Omega$; cf. [6, proof of (3.4), pp. 96, 99]. This completes the proof of Theorem A.

\section{REFERENCES}

1. A. D. Alexandrow, Die innere Geometrie der konvexen Flächen, Academie-Verlag, Berlin, 1955.

2. P. Hartman, On the local uniqueness of geodesics, Amer. J. Math. 72 (1950), 723-730.

3. On geodesic coordinates, Amer. J. Math. 73 (1951), 949-954.

4. - On exterior derivatives and solutions of ordinary differential equations, Trans. Amer. Math. Soc. 91 (1959), 277-293. 
5. On uniqueness and differentiability of solutions of ordinary differential equations, Nonlinear Problems (R. E. Langer, ed.), (Proc. Sympos., Madison, Wis., 1962), Univ. of Wisconsin Press, Madison, Wis., 1963, pp. 219-232.

6. __ Ordinary differential equations, Birkhäuser, Boston, Mass., 1982.

7. P. Hartman and A. Wintner, On the problems of geodesics in the small, Amer. J. Math. 73 (1951), $132-148$.

8. __ On the third fundamental form of a surface, Amer. J. Math. 75 (1953), 298-334.

9. H. Petrini, Les dérivées premières et secondes du potentiel logarithmique, J. de Math. 5 (1909), 127-223.

MONTEREY, CALIFORNIA 